\title{
sciendo THE ASSEMBLY LOADS AND SOME STRENGTHENING METHODS OF THE PRODUCTS FROM POROUS MATERIAL WITH COILED WIRE
}

DOI 10.2478/ntpe-2018-0082

\author{
Dr. Andrzej Jakubowski \\ Maritime University of Szczecin, Poland
}

2018

Volume 1

Issue 1

pp. $655-660$

\begin{abstract}
The product from porous permeable material based on a wire coil consists of a round, porous, thin-walled tube. The impact of assembly loads of the porous tube has been examined to determine the maximum values of the compressive force and axial elastic strain during the assembly. To improve mechanical properties of the product additional reinforcement has been proposed: larger diameter wire, woven wire cloth or spring.
\end{abstract}

Keywords: wire, porous tube, assembly loads, reinforcement

\section{INTRODUCTION}

Among numerous porous materials (PM) (Belov, 1987), the most efficient materials in terms of dynamic permeability and dynamic strength are those using woven and knotted nets (Sinelnikov et al.,1983). A relatively new PM with a similar structure is referred to as porous material with coiled wire (CWPM) (Petyushik et al., 2001). Such material is composed of crossed and layered wire wound on a cylindrical holder, subsequently subjected to deformative processing, i.e. radial reduction. This technology excludes thermal treatment - sintering essential in the manufacture of powder materials and several fibrous materials. In this way CWPMs acquire the form of a round, porous, thin-walled tube with length $L$ and internal diameter $D_{\text {opr }}$, composed of wire with constant diameter $d$, which is placed in $n$ number of layers at angle $\beta$ and constant spacing $s$. The structure is regarded as regular throughout the material volume.

An important question in using CWPM products is how assembly loads affect the structure of the material and its properties. During its installation a porous tube gets compressed along its axis (Fig. 1).

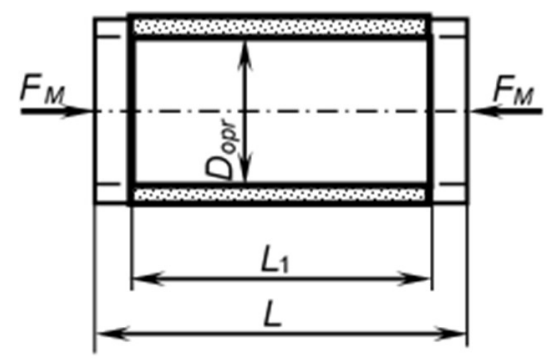

Fig. 1. The diagram illustrating assembly loads acting on a CWPM product.

The condition for maintaining the tube strength is that it can be compressed within the limits of elastic deformation, so that the skeleton stiffness is preserved without affecting its structure, while the fixed relative distribution of wire coil turns is deformed (Liu et al., 2009).

High efficiency of modern machinery is often directly related to the occurrence of high dynamic loads that may affect work safety or equipment failure. CWPM-containing objects such as porous tubes, characterized by anisotropy of mechanical properties, have a maximum strength 
in the direction of wire coil turns, i.e. tangentially. The direction of assembly loads is different, i.e. axial, while the direction of operational loads is radial. The use of wire products calls for the compensation of their anisotropic properties and for providing them with some specific properties. This can be achieved, for example, by reinforcement (Kim et al., 1991).

\section{ASSEMBLY LOADS}

The external assembly force is transferred through the coil wire contact areas inside the skeleton (Fig. 2) throughout the tube volume (Yakubouski and Yakubouski, 2005). The overall axial assembly load is equal to:

$$
F_{M}=n_{\kappa} F_{1},
$$

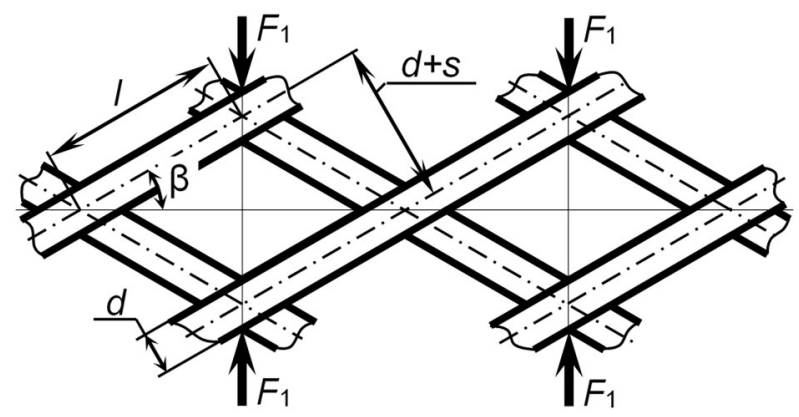

Fig. 2. A diagram of cell load in the CWPM skeleton during tube assembly

where:

$F_{1}$ - force in one contact area;

$n_{K}-$ number of contact areas, determined as (Jakubowski and Crasto, 2016):

$$
n_{\kappa}=\frac{2 \pi n L\left(D_{o p r}+h\right) \sin 2 \beta}{(d+s)^{2}} .
$$

An organized CWPM structure allows presenting a material skeleton cell as a closed circuit in the form of a truss with links of length $l$, loaded in the nodes by concentrated forces $F_{1}$ (Fig. $3 a)$. The truss should be considered as an unhinged system due to the stiffness of contact nodes obtained through plastic deformation in the process of CWPM manufacturing. Such a structure is three times statically indeterminate, but conditions of system symmetry and load symmetry permit to reduce the number of unknowns to one (Feodosyev, 1979). Thus, selecting a calculation scheme we cut off the circuit along the horizontal axis of symmetry I-I. In the cross-section $I-I$ transverse and longitudinal internal forces are found from the condition of equilibrium (Fig. 3b):

$$
Q=\frac{F_{1} \cos \beta}{2} ; \quad N=-\frac{F_{1} \sin \beta}{2} .
$$

To solve the static indeterminacy of the truss, we combine the canonic equation of the method of forces (Feodosyev, 1979):

$$
\delta_{11} X_{1}+\Delta_{1 F}=0 .
$$

We calculate the coefficient $\delta_{11}$ with unnecessary, unknown moment $X_{1}$ and free term $\Delta_{1 F}$ of equation (4) according to the A.N Vereshchagin's rule (Feodosyev, 1979) by multiplying the curve of load moments (Fig. 3c) with the curve of unit moments (Fig. 3d), then from the equation (4) we express the unknown $X_{1}$ :

$$
X_{1}=-\frac{\left(\frac{1}{2} \cdot \frac{F_{1} l \cos \beta}{2} \cdot l \cdot 1\right) \cdot 2}{(1 \cdot l \cdot 1) \cdot 2}=-\frac{F_{1} l \cos \beta}{4},
$$

and finally we draw a curve of bending moments $M$ for the whole circuit (Fig. 3e). 

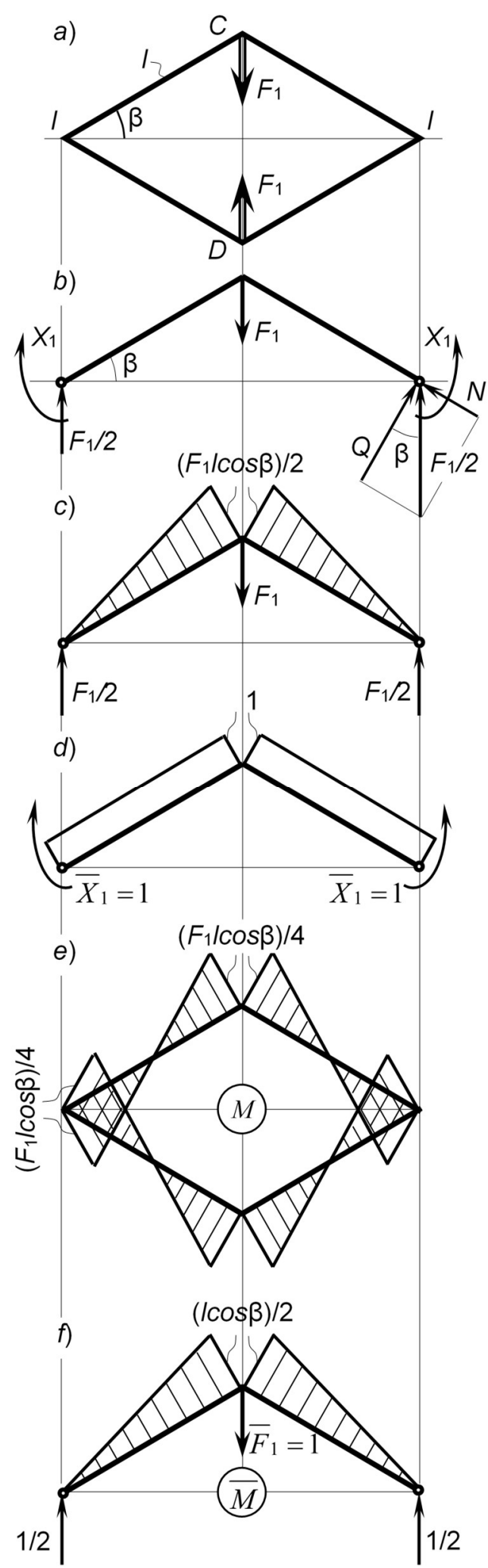

Fig. 3. Graphic part of calculation (curves) of the closed circuit as an unhinged truss The links in the truss are subject to bending and compression.

Normal stresses in dangerous cross-sections of the truss during bending and compression of the links equal, respectively (Feodosyev, 1979):

$$
\sigma_{u}=\frac{M}{W}=\frac{8 F_{1} l \cos \beta}{\pi d^{3}} ; \quad \sigma_{c}=\frac{N}{A}=\frac{2 F_{1} \sin \beta}{\pi d^{2}},
$$

where:

$W=\pi d^{3} / 32-$ the wire cross-section modulus. 
Total normal stresses are determined as follows:

$$
\sigma=\sigma_{u}+\sigma_{c}=\frac{2 F_{1}}{\pi d^{2}} \cdot\left(\frac{4 l}{d}+\operatorname{tg} \beta\right) \cdot \cos \beta
$$

Taking into account previously mentioned recommendations for improving the bonding of wire turns by increasing contact areas and deflections (the wire should be coiled at a small angle in the range of $\left.5^{\circ} \ldots 45^{\circ}\right)$, it is clear that in the (7)

$$
\frac{\sigma_{c}}{\sigma_{u}}=\frac{d}{4 l} \cdot \operatorname{tg} \beta=\frac{\sin ^{2} \beta}{2 \cdot(1+s / d)}<<1
$$

Consequently, comparing the normal stress to the yield point of the wire material, from expression (7) we get:

$$
\sigma=\frac{8 F_{1} l \cos \beta}{\pi d^{3}}=\sigma_{p l}
$$

Hence we determine the maximum compressive load in the closed circuit:

$$
F_{1}=\frac{\pi d^{3} \sigma_{p l}}{8 l \cos \beta}=\frac{A \sigma_{p l} \sin \beta}{1+s / d}
$$

Equation (1), taking into account the expressions (2) and (10), will eventually get this form:

$$
F_{M}^{\max }=A_{r} \sigma_{p l} \cdot \frac{L \sin \beta \sin 2 \beta}{(d+s)^{3} / A},
$$

where:

$A_{r}=\pi H\left(D_{\text {opr }}+h\right)-$ area of the load of the tube cross-section.

To determine the deformation of porous tube during its assembly we should also consider the material skeleton cell, which is done by calculating the distance $\delta_{C D}$ between points $C$ and $D$ of the closed circuit, equal to double displacement of one of these points, for example $\delta_{c}$. According to Vereshchagin's rule (Yakubouski, A.Ch., Yakubouski, Ch.A. 2005), by multiplying the curve of bending moments (see Fig. $3 e$ ) with the curve of unit moments (Fig. 3f), we get:

$$
\delta_{C D}=2 \delta_{C}=2 \cdot \frac{1}{E I} \cdot\left(\frac{1}{2} \cdot l \cdot \frac{F_{1} l \cos \beta}{4} \cdot \frac{2}{3} \cdot \frac{l \cos \beta}{2}-\frac{1}{2} \cdot l \cdot \frac{F_{1} l \cos \beta}{4} \cdot \frac{1}{3} \cdot \frac{l \cos \beta}{2}\right) \cdot 2=\frac{F_{1} l^{3} \cos ^{2} \beta}{12 E I} .
$$

Then the maximum elastic strain of the whole tube equals:

$$
\Delta_{M}^{\max }=\frac{F_{M}^{\max } l^{3} \cos ^{2} \beta}{12 E I}=\frac{A_{r} L}{3 d^{2} \sin \beta} \cdot \frac{\sigma_{p l}}{E} .
$$

Expressions (11) and (13) describe the state of stresses and strains of the CWPM skeleton, resulting from the porous tube assembly, and determie the critical compressive forces and elastic deformations, depending on tube size, the material and size of wire used, and technological conditions.

\section{MATERIAL STRENGTHENING METHODS}

Reinforcement is made at the stage of forming wire coil (Fig. 4) by placing reinforcing elements on the inside, outside or between the material layers (Toll, 1998). The quantity and distribution of reinforcement are determined according to expected conditions of operation. Notably, the reinforcement does not change the object permeability (if it should specifically be assured), because the volume occupied by the reinforcement is relatively small. There are various possible methods of reinforcement.

1. Reinforcement made of wire with a larger diameter or metal rods (Yakubouski et al., 2011):

a) longitudinal (Fig. 4a) - reinforcing wires, located along the cylindrical surface of a porous tube, permits to increase significantly the tube strength axially (useful during installation), but it does not resist radial pressure;

b) spiral (Fig. 4b) - reinforcement in the form of unidirectional spiral wire will make the tube resist radial forces, but does not provide for increased axial strength, because spirals, when compressed during assembly, additionally cause torsional stresses; 


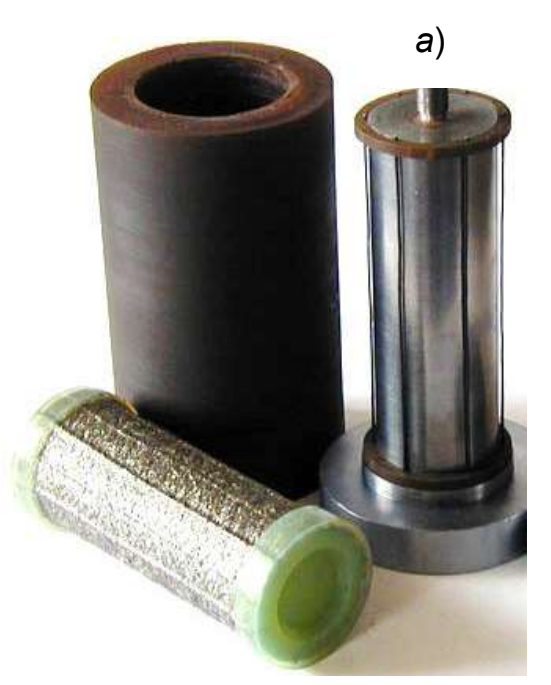

b)

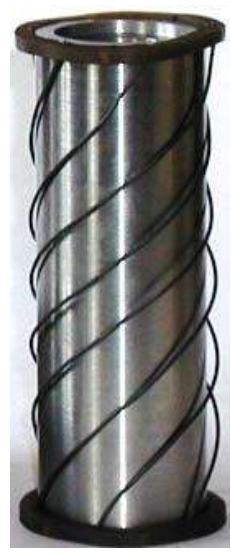

c)

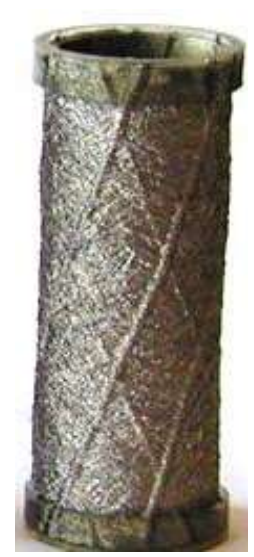

d)

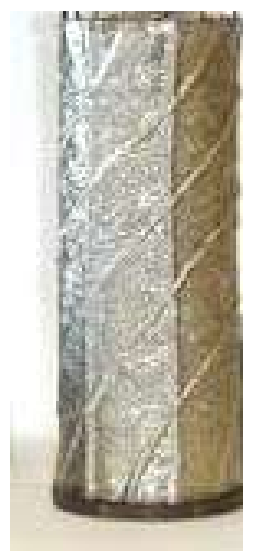

Fig. 4. Various forms of CWPM reinforcement with larger wire diameter: longitudinal (a), spiral (b), spiral reversed (c), combined (d)

c) reversed spirals (Fig. 4c) - reinforcement in the form of two-directional spiral wires strengthen the tube radially and axially;

d) combined (Fig. 4d) - reinforcement in the form of straight wires together with wire spirals, in many cases may be the most efficient method.

2. Reinforcement by a spiral spring (Fig. 5a) increases the strength and stiffness of the material radially and adds flexibility to the object.

3. Reinforcement made of thick woven mesh (Fig. $5 b$ ) provides high strength and stiffness of the material in all directions.

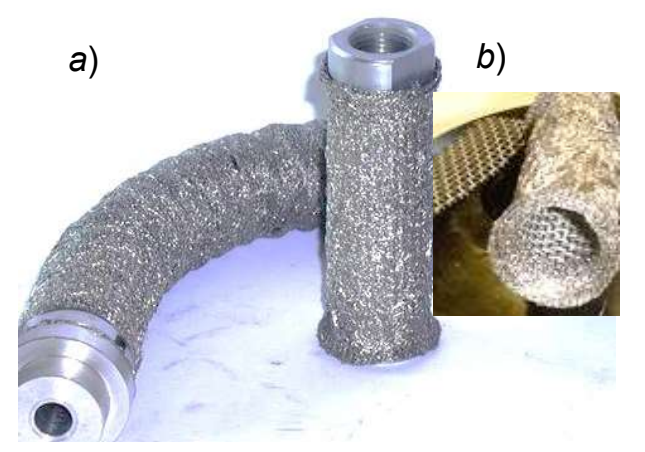

Fig. 5. CWPM reinforcement: spring (a) and woven mesh (b)

\section{CONCLUSIONS}

The study of stresses and strains of the porous tube during its assembly was based on the concept of the material skeleton cell represented by a closed circuit - an unhinged truss, loaded at the nodes by concentrated force. The authors also established the direct influence of wire size and properties on maximum values of the compressive force and axial deformation. In order to increase the mechanical properties of CWPM products we have proposed additional reinforcement made of larger diameter wire, woven mesh or springs.

\section{REFERENCES}

Belov, S.V. (1987). Poristyye Pronitsayemyye Materialy. Metallurgiya, 335.

Feodosyev, V.I. (1979). Soprotivlenie Materialov. Nauka, 560.

Jakubowski, A., Crasto, R. (2016). The Structure Analytical Research of Porous Permeable Wire Material. Management Systems in Production Engineering, V. 2(22), pp. 81-88. 
Kim, Y., Mccarthy, S., Fanucci, J. (1991). Compressibility and Relaxation of Fiber Reinforcements During Composite Processing. Polymer Composites, 12(1), pp. 13-19.

Liu, P., He, G., Wu, L. (2009). Structure Deformation And Failure Of Sintered Steel Wire Mesh Under Torsion Loading. Materials \& Design, 30(6), pp. 2264-2268.

Petyushik, Ye.Ye., Reut, O.P., Yakubouski, A.Ch., Boginskiy L.S. (2001). Main Aspects of the Theory and Technology of Producing Permeable Materials with the Organized Porous Structure Through Deformation Processing. Reutte (Austria): 15 International Plansee Seminar, V. 3, pp. 285-299.

Sinelnikov, Yu.l. et al. (1983). Poristyye Setchatyye Materialy. Metallurgiya, 63.

Toll, S. (1998). Packing Mechanics of Fiber Reinforcements. Polymer Engineering and Science, 38(8), pp. 1337-1350.

Yakubouski, A.Ch., Yakubouski, Ch.A. (2005). Raschet Montazhnyh Nagruzok v Poristom Provolochnom Izdelii. Minsk: Teoreticheskaya i prikladnaya mechanika, V. 18, pp. 118121.

Yakubouski, A.Ch., Yakubouski, Ch.A., Galuza, I.M., Galuza, E.M. (2011). Armirovanie Poristogo Materiala na Osnove Navivaemoy Provoloki dla Ispolzovania v Kachestve Dempfiruyushchih Elementov. Minsk: Mashinostroenie, V. 26(2), pp. 177-179.

Date of submission of the article to the Editor: 02/2018

Date of acceptance of the article by the Editor: 05/2018 